Pacific Journal of Mathematic 


\section{THE POLYNOMIAL HULLS OF CERTAIN SUBSETS OF $\boldsymbol{C}^{2}$}

T. W. GaMelin

Summary. Fix $\alpha \leqq 0$. We will describe the polynomial hulls of compact subsets of $C^{2}$ that are invariant under the transformations $T_{\theta}, 0 \leqq \theta \leqq 2 \pi$, defined by

$$
T_{\theta}(z, w)=\left(e^{i \theta} z, e^{i \alpha \theta} w\right), \quad(z, w) \in C^{2} .
$$

1. Introduction. Let $X$ be a compact subset of $C^{2}$. In [10], J. Wermer describes the polynomial hull $\hat{X}$ of $X$, for a certain class of sets $X$ which are invariant under the one-parameter group of transformations

$$
(z, w) \longrightarrow\left(e^{i \theta} z, e^{-i \theta} w\right), \quad 0 \leqq \theta \leqq 2 \pi
$$

He develops an idea for introducing analytic structure in $\hat{X} \backslash X$, and he shows in particular that every point of $\hat{X} \backslash X$ lies on an analytic $\operatorname{disc}$ in $\hat{X}$.

Our aim is to combine Wermer's ideas with some elementary results in potential theory, in order to describe the polynomial hull $\hat{X}$ of an arbitrary compact subset $X$ of $C^{2}$ invariant under the group (1.1), or more generally under the transformations defined by (0.1).

If the number $\alpha$ is irrational, then the transformation group defined by (0.1) is dense in the two-parameter group of transformations

$$
(z, w) \longrightarrow\left(e^{i \theta} z, e^{i \varphi} w\right), \quad 0 \leqq \theta, \varphi \leqq 2 \pi
$$

If $X$ is invariant under the group $(0,1)$, then $X$ is also invariant under the groups (1.2). Such sets are said to be circled. The description of the polynomial hull of compact circled sets is classical ([6], [2], [3], [4, §III. 3], [7, §2.4], [9, §14]). We may confine our attention to the case in which $\alpha$ is rational.

Consider next the case $\alpha=0$. The transformations (0.1) then assume the form

$$
(z, w) \longrightarrow\left(e^{i \theta} z, w\right), \quad 0 \leqq \theta \leqq 2 \pi
$$

If $X$ is invariant under the transformations (1.3), its polynomial hull can be described as follows [9]. Let $J$ be the projection of $X$ into the $w$-plane, and define

$$
r(w)=\sup \{|z|:(z, w) \in X\}, \quad w \in J .
$$

Define a function $R$ on the polynomial hull $\hat{J}$ of $J$ by requiring that $\log R$ be the lower envelope of the family of functions $u$ which are 
superharmonic in a neighborhood of $\hat{J}$ and which satisfy $u \geqq \log r$ on $J$. Then

$$
\hat{X}=\{(z, w): w \in \hat{J},|z| \leqq R(w)\} .
$$

(This description is valid for subsets of $C^{n}$, providing $w$ is interpreted as an $(n-1)$-tuple in $C^{n-1}$, and "superharmonic" is replaced by "plurisuperharmonic".)

In $\S 5$ we formulate our main result, Theorem 5.2, which describes $\hat{X}$ in the case that $\alpha=-1$, that is, in the case that $X$ is invariant under the group (1.1). The description again involves the envelopes of certain families of superharmonic functions.

Sections 2 through 4 include some preliminary results, while the proof of Theorem 5.2 is given in $\S \S 6$ and 8. In $\S 9$, we indicate how the discussion can be modified to cover the case in which $\alpha$ is an arbitrary negative rational number. In $\$ 10$ we observe that every point of $\hat{X} \backslash X$ lies on an analytic disc in $\hat{X}$, and that $P(X)=C(X)$ whenever there are no analytic discs in $\hat{X}$.

A source for standard definitions and notation is [4]. The complex plane will be denoted by $C$, and the open disc with center $z$ and radius $\delta$ will be denoted by $\Delta(z ; \delta)$.

The space of all continuous complex-valued functions on $X$ is denoted by $C(X)$. The uniform closure in $C(X)$ of the analytic polynomials is denoted, as usual, by $P(X)$. Then $\hat{X}$ can be identified with the maximal ideal space of $P(X)$, and $P(X)$ is isometrically isomorphic to $P(\hat{X})$.

2. A result from potential theory. Let $K$ be a compact subset of $C$, such that $C \backslash K$ is connected. Let $\phi$ be an upper semicontinuous function from $K$ to $[-\infty, \infty)$. Let $\mathscr{Q}$ be the family of real-valued functions $\psi$ which are continuous and superharmonic on a neighborhood of $K$, and which satisfy $\psi \geqq \varphi$ on $K$. Let $\Psi$ be the lower envelope of $Q$ :

$$
\Psi(z)=\inf \{\psi(z): \psi \in \mathbb{Q}\}
$$

Then $\Psi$ is an upper semi-continuous function from $K$ to $[-\infty, \infty)$ which satisfies

$$
\varphi(z) \leqq \Psi(z)
$$$$
z \in K \text {. }
$$

THEOREM 2.1. Let $K, \varphi$ and $\psi$ be as above. Let $\Omega$ be the subset of $z \in K$ such that $\varphi(z)<\Psi(z)$. Then $\Omega$ is an open subset of $C$, and $\Psi$ is harmonic on $\Omega$.

Proof. Since $\phi$ is upper semi-continuous, there exists $c>0$ such 
that

$$
\varphi(z)<c, \quad z \in K \text {. }
$$

Then also $\Psi(z)<c$ for all $z \in K$.

First we show that $\varphi\left(z_{0}\right)=\Psi\left(z_{0}\right)$ if $z_{0} \in \partial K$. This depends only on the existence of a barrier at $z_{0}$, and the proof is classical. Indeed, let $\varepsilon>0$, and let $N$ be a neighborhood of $z_{0}$ such that $\varphi(z)<\varphi\left(z_{0}\right)+\varepsilon$ for $z \in N \cap K$. Since $z_{0}$ is a regular point for the "outer" Dirichlet problem (or since $z_{0}$ is a peak point for $P(K)$ ), there exists a function $u$ harmonic in a neighborhood of $K$, such that $u>0$ on $K, u\left(z_{0}\right)<\varepsilon$, and $u(z)>c-\varphi(z)$ for $z \in K \backslash N$. The function $\psi=u+\psi\left(z_{0}\right)$ is then harmonic in a neighborhood of $K, \psi>\varphi$ on $K$, and $\psi\left(z_{0}\right)<\varphi\left(z_{0}\right)+\varepsilon$. It follows that $\Psi\left(z_{0}\right)<\varphi\left(z_{0}\right)+\varepsilon$, this for all $\varepsilon>0$, so that

$$
\Psi\left(z_{0}\right)=\varphi\left(z_{0}\right), \quad z_{0} \in \partial K .
$$

We have shown that $\Omega$ is a subset the interior of $K$.

Next we wish to show that $\Omega$ is open. We will use the following standard estimate, which follows for instance from [8, Theorem III. $67]$.

LEMMA 2.2. There is a positive function $\chi(t), t>0$, such that $\chi(t) \rightarrow 0$ as $t \rightarrow 0$, which has the following property. If $\Delta_{0}=\Delta\left(\zeta_{0} ; \delta\right)$ is any open disc, if $W$ is a domain such that $\zeta_{0} \in W \subset \Delta_{0}$, if $E$ is a connected subset of $\bar{\Delta}_{0} \mid W$ which meets $\partial \Delta_{0}$, and if $d=\inf \left\{\left|\zeta-\zeta_{0}\right|:\right.$ $\zeta \in E\}$, then the harmonic measure $\mu_{\xi_{0}}$ on $\partial W$ for $\zeta_{0}$ satisfies

$$
\mu_{\zeta_{0}}\left(\partial \Delta_{0} \cap \partial W\right) \leqq \chi(d / \delta)
$$

Now let $\zeta_{0} \in \Omega$. Choose $a$ so that $\varphi\left(\zeta_{0}\right)<a<\Psi\left(\zeta_{0}\right)$. On account of the upper semi-continuity of $\varphi$, it suffices to show that $\Psi(\zeta) \geqq a$ for $\zeta$ near $\zeta_{0}$. For this, it suffices to find $\delta_{0}>0$ such that $\psi(\zeta) \geqq \alpha$ for all $\zeta \in \Delta\left(\zeta_{0} ; \delta_{0}\right)$ and for all functions $\psi$ which are continuous and superharmonic in a neighborhood of $K$ and satisfy $\psi \geqq \varphi$ on $K$.

So let $\psi$ be such a function. Replacing $\psi$ by $\min (\psi, c)$, we can assume that $\psi \leqq c$.

Let $\Delta_{0}=\Delta\left(\zeta_{0} ; \delta\right)$ be an open disc such that $\bar{\Delta}_{0} \subset K^{0}$, and such that

$$
\varphi(\zeta)<a, \quad \zeta \in \bar{\Delta}_{0} .
$$

The set $\left\{\zeta \in \Delta_{0}: \psi(\zeta)>a\right\}$ is open and includes $\zeta_{0}$. Let $W$ be the component of this set which includes $\zeta_{0}$. Then $\psi=a$ on $\Delta_{0} \cap \partial W$, while $\psi \geqq a$ on $\partial \Delta_{0} \cap \partial W$.

We claim that every component of $\overline{\Delta_{0}} \backslash W$ meets $\partial \Delta_{0}$. Indeed, sup- 
pose not. Then there is a connected open subset $U$ of $\Delta_{0}$ such that $\partial U \subset W$, and $U$ includes a portion of $\partial W$. Now $U \cup W$ is connected, and $\partial(U \cup W) \subset \partial W$, so that $\psi \geqq a$ on $\partial(U \cup W)$. Since $\psi$ attains its minimum value $a$ over $U \cup W$ on the portion of $\partial W$ inside $U \cup W, \psi$ is identically equal to $a$ on $U \cup W$. This contradicts the definition of $W$, and the claim is established.

Let $\mu_{\zeta}$ be the harmonic measure on $\partial W$ for $\zeta \in W$. Define

$$
\psi_{1}(\zeta)=\left\{\begin{array}{l}
\psi(\zeta), \\
\int_{\partial W} \psi d \mu_{\zeta},
\end{array}\right.
$$

On account of the preceding paragraph, every point of $\partial W$ is a regular point for the Dirichlet problem, so that $\psi_{1}$ is continuous in a neighborhood of $K$. Evidently $\psi_{1}$ is superharmonic. Since $\psi_{1} \geqq a$ on $\partial W$, also $\psi_{1} \geqq a$ on $W$. Consequently $\psi_{1} \geqq \varphi$, and $\psi_{1} \in \mathbb{Q}$.

Now

$$
\Psi\left(\zeta_{0}\right)-a \leqq \psi_{1}\left(\zeta_{0}\right)-a=\int_{\partial W}[\psi(\zeta)-a] d \mu_{\zeta_{0}}(\zeta)
$$

Since $\psi=a$ on $\Delta_{0} \cap \partial W$, the integral is bounded above by $(c-a) \mu_{\xi_{0}}\left(\partial \Delta_{0} \cap \partial W\right)$. The estimate of Lemma 2.2 then yields

$$
\Psi\left(\zeta_{0}\right)-a \leqq(c-a) \chi(d / \delta),
$$

when $d$ is the distance from $\zeta_{0}$ to $\partial W$. Since $c>a$ and $\Psi\left(\zeta_{0}\right)>a$, the quantity $\chi(d / \delta)$ is bounded away from zero, independent of $\psi$. Consequently the quantity $d$ is bounded away from zero, independent of $\psi$. Let $\delta_{0}>0$ be a lower bound for such $d$. From the definition of $d$, we obtain $\Delta\left(\zeta_{0} ; \delta_{0}\right) \subset W$, hence $\psi>a$ on $\Delta\left(\zeta_{0} ; \delta_{0}\right)$, this for all $\psi$, so that $\Psi \geqq a$ on $\Delta\left(\zeta_{0} ; \delta_{0}\right)$. We conclude that $\Delta\left(\zeta_{0} ; \delta_{0}\right) \subset \Omega$, and $\Omega$ is open.

Now the restrictions of the functions in $Q$ to $\Omega$ form a Perron family on $\Omega$. The classical proof then shows that their lower envelope $\Psi$ is either harmonic on $\Omega$ or is equal to $-\infty$ on $\Omega$. Since $\Psi>\varphi$ on $\Omega$, the latter alternative is excluded, and the theorem is proved.

3. Analytic discs. The maximum modulus principle allows us to conclude that if $V$ is an analytic variety in $C^{n}$ whose boundary lies in $X$, then $V \subset \hat{X}$. We will need a slightly more general assertion to the same effect.

A subset $S$ of $C^{n}$ is an analytic disc if there is a continuous one-to-one map $\varphi$ of an open disc $\Delta_{0}$ in the complex plane onto $S$, such that $f \circ \varnothing$ is analytic on $A_{0}$ for every analytic polynomial $f$ on $C^{n}$. 
Lemma 3.1. Let $Y$ be a compact subset of $C^{n}$. Let $E$ be a bounded subset of $C^{n}$ such that $\bar{E} \backslash E \subset Y$, and such that $E$ is a union of analytic discs. Then $E \subset \hat{Y}$.

Proof. Let $y \in Y \cup E$ be a peak point for $P(Y \cup E)$. Then $y$ can lie on no analytic disc in $Y \cup E$; otherwise any function peaking at $y$ would be constant on that disc, an absurdity. Consequently the peak points for $P(Y \cup E)$ all lie in $Y$. Since these are dense in the Shilov boundary, the Shilov boundary of $P(Y \cup E)$ is contained in $Y$. It follows that $E \subset \hat{Y}$.

4. A lemma of B. Cole. The next lemma is due to by $B$. Cole, who uses it in his work extending the classical inequalities of A. N. Kolmogoroff, M. Riesz and A. Zygmund to a function-algebra setting ${ }^{1}$.

LEMMA 4.1. Let $J$ be a compact subset of $C$. Let $\zeta_{0} \in \widehat{J}$, and let $\eta$ be a measure on $J$ which is a Jensen measure for $\zeta_{0}$ with respect to the algebra $P(J)$. Then

$$
\int \psi d \eta \leqq \psi\left(\zeta_{0}\right)
$$

for any real-valued function \& which is superharmonic in a neighborhood of $\hat{J}$.

Proof. Recall that a Jensen measure for $\zeta_{0}$ is a probability measure $\eta$ on $J$ which satisfies

$$
\log \left|f\left(\zeta_{0}\right)\right| \leqq \int \log |f| d \eta
$$

for all $f \in P(J)$. In particular,

$$
\log \left|\zeta_{0}-\xi\right| \leqq \int \log |\zeta-\xi| d \eta(\zeta), \quad \xi \in C
$$

By a theorem of F. Riesz, $\psi$ is the logarithmic potential of some positive measure $\lambda$ defined in a neighborhood of $\hat{J}$ :

$$
\psi(\zeta)=-\int \log |\zeta-\xi| d \lambda(\xi), \quad \zeta \in \hat{J} .
$$

Integrating with respect to $\eta$, interchanging the orders of integration, and using (4.1), we obtain

$$
\int \psi(\zeta) d \eta(\zeta) \leqq-\int \log \left|\zeta_{0}-\xi\right| d \lambda(\xi)=\psi\left(\zeta_{0}\right),
$$

${ }^{1}$ Communicated to a Colloquium audience at Tulane University, December, 1970. 
as required.

5. Formulation of the theorem.

In $\$ \S 5$ through 8 we assume that $X$ is a compact subset of $C^{2}$ that is invariant under the transformations

Define $F: \hat{X} \rightarrow C$ by

$$
T_{\theta}:(z, w) \longrightarrow\left(e^{i \theta} z, e^{-i \theta} w\right), \quad 0 \leqq \theta \leqq 2 \pi .
$$

$$
F(z, w)=z w, \quad(z, w) \in \hat{X} .
$$

The fiber $M_{\zeta}$ of $\hat{X}$ over $\zeta$ is defined by

$$
M_{\zeta}=F^{-1}(\zeta), \quad \zeta \in F(\hat{X}) .
$$

The various $M_{\zeta}$ partition $\hat{X}$ into disjoint closed subsets. Evidently $\hat{X}$ is invariant under the $T_{\theta}$, and one checks easily that each $M_{\zeta}$ is invariant under the $T_{\theta}$. For $\zeta \in F(\hat{X})$, define

$$
\begin{aligned}
& Q(\zeta)=\sup \left\{|z|:(z, w) \in M_{\zeta}\right\}, \\
& R(\zeta)=\sup \left\{|w|:(z, w) \in M_{\zeta}\right\} .
\end{aligned}
$$

Since $z w=\zeta$ on $M_{\zeta}$,

$$
\inf \left\{|z|:(z, w) \in M_{\zeta}\right\}=|\zeta| / R(\zeta),
$$

for $\zeta \in F(\hat{X}) \backslash\{0\}$. In particular; the infimum is strictly positive, for $\zeta \neq 0$.

Lemma 5.1. If $\zeta \in F(\hat{X}), \zeta \neq 0$, then $M_{\zeta}$ coincides with the circle or closed annulus described by

$$
\left\{(z, \zeta / z): \frac{|\zeta|}{R(\zeta)} \leqq|z| \leqq Q(\zeta)\right\}
$$

Furthermore, if $0 \in F(\hat{X})$, then $M_{0}$ coincides with the set

$$
\{(z, 0):|z| \leqq Q(0)\} \cup\{(0, w):|w| \leqq R(0)\} .
$$

Proof. Suppose $\zeta \neq 0$. On account of (5.5) and the definitions of $Q$ and $R, M_{\zeta}$ is included in the annulus (5.6). Furthermore, on account of the invariance of $M_{\zeta}$ under the $T_{\theta}, M_{\zeta}$ includes the boundary circles of (5.6). Since the annulus described by (5.6) is "analytic", $M_{\zeta}$ includes the entire annulus. That proves the first statement of the lemma, and the proof of the second statement is similar.

To describe $\hat{X}$, it now suffices to determine $F(\hat{X})$, and to determine 
the upper semi-continuous functions $Q(\zeta)$ and $R(\zeta)$. Since the problem at hand is invariant under an interchange of coordinates, any expression for $Q(\zeta)$ will lead to an expression for $R(\zeta)$. Consequently it suffices to describe $F(\hat{X})$ and $Q$.

Let $q$ be the upper semi-continuous function on $F(X)$ defined by

$$
\begin{aligned}
q(\zeta) & =\sup \left\{|z|:(z, w) \in X \cap M_{\zeta}\right\} \\
& =\sup \{|z|:(z, \zeta / z) \in X\} .
\end{aligned}
$$

Let $K$ be the polynomial hull of $F(X)$ :

$$
K=\widehat{F(X)} \text {. }
$$

Let $Q$ be the family of real-valued functions $\psi$ such that $\psi$ is superharmonic in a neighborhood of $K$ and $\psi \geqq \log q$ on $F(X)$. Define

$$
\Psi(\zeta)=\inf \{\psi(\zeta): \psi \in \mathscr{Q}\}
$$$$
\zeta \in K \text {. }
$$

The following theorem yields the description of $\hat{X}$ that we are aiming at.

THEOREM 5.2. Let $X$ be a compact subset of $\boldsymbol{C}^{2}$ that is invariant under the $T_{\theta}$ given by (5.1). Let $F, Q$ and $\psi$ be as defined above. Then $F(\hat{X})$ coincides with the polynomial hull $K$ of $F(X)$. Furthermore,

$$
Q(\zeta)=\exp (\Psi(\zeta))
$$

The proof will be postponed to $\S 8$. Here we make two preliminary observations.

Lemma 5.3. $F(\hat{X})$ is included in $K=\widehat{F(X)}$.

Proof. This follows from an elementary principle in Banachalgebra theory, since $F \in P(X)$.

LEMmA 5.4. If $\zeta \in F(\hat{X})$, then

$$
Q(\zeta) \leqq \exp (\Psi(\zeta))
$$

Proof. Let $\zeta_{0} \in F(X)$, and let $\left(z_{0}, w_{0}\right) \in M_{\zeta_{0}}$. Let $\nu$ be a Jensen measure on $X$ for $\left(z_{0}, w_{0}\right)$ with respect to the algebra $P(X)$. Then the projected measure $F^{*}(\nu)$ is a Jensen measure on $F(X)$ for $\zeta_{0}$ with respect to the algebra $P(K)$. For any $\psi \in \mathscr{Q}, \log \left|z_{0}\right| \leqq \int \log |z| d \nu(z$, $w) \leqq \int \log q(\zeta) d\left(F^{*} \nu\right)(\zeta) \leqq \int \psi(\zeta) d\left(F^{*} \nu\right)(\zeta)$. By Cole's lemma, the latter integral is bounded by $\psi\left(\zeta_{0}\right)$. Taking the infimum over $\psi$ and the supremum over $\left(z_{0}, w_{0}\right) \in M_{\xi_{0}}$, we obtain (5.12). 
6. Proof of Theorem 5.2: A special case. In this section, we assume that

$$
F(X)=\partial K,
$$

that is, $F(X)$ forms the boundary of its polynomial hull. In this case, the lower envelope $\Psi$ of $Q$ is given by

$$
\Psi(\zeta)= \begin{cases}\log q(\zeta), & \zeta \in \partial K, \\ \int \log q(\xi) d \mu_{\zeta}(\xi), & \zeta \in K^{0},\end{cases}
$$

where $\mu_{\zeta}$ is the harmonic measure on $\partial K$ for $\zeta \in K^{0}$. We will show shortly that the integral converges.

Define a lower semi-continuous function $p$ on $\partial K$ by

$$
p(\zeta)=\inf \left\{|z|:(z, w) \in X \cap M_{\zeta}\right\}, \quad \zeta \in \partial K .
$$

Then $-\infty \leqq p \leqq q$. Let $c>0$ satisfy

$$
|z|,|w| \leqq c, \quad(z, w) \in X .
$$

The relation $z w=\zeta$ then yields the estimate

$$
\log |z| \geqq \log |\zeta|-\log c, \quad(z, w) \in X \cap M_{\zeta} .
$$

Taking an infimum, we obtain

$$
\log p(\zeta) \geqq \log |\zeta|-\log c, \quad \zeta \in \partial K .
$$

A simple application of Fatou's Lemma reveals that the function $\log |\zeta|$ is integrable with respect to harmonic measure on $\partial K[1, \mathrm{p}$. 170]. Consequently $\log p$ and $\log q$ are both integrable with respect to $\mu_{\zeta}$. In particular, $\Psi$ is finite.

Define

$$
\Phi(\zeta)= \begin{cases}\log p(\zeta), & \zeta \in \partial K, \\ \int \log p(\xi) d \mu_{\zeta}(\xi), & \zeta \in K^{0} .\end{cases}
$$

Then $\Phi(\zeta)>-\infty$ on $K^{0}$, and

$$
\Phi(\zeta) \leqq \Psi(\zeta), \quad \zeta \in K
$$

Since each component of $K^{0}$ is simply connected, every point of $\partial K^{0}$ is a regular point for the Dirichlet problem, and the mass of $\mu_{\xi}$ accumulates towards $\xi$ as $\zeta \in K^{0}$ tends to $\xi \in \partial K$. On account of the semi-continuity of $\log p$ and $\log q$, we obtain

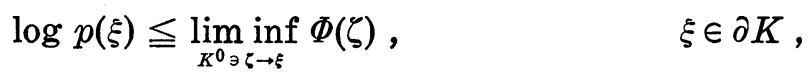




$$
\log q(\xi) \geqq \lim _{K^{0} \ni \zeta \rightarrow \xi} \sup _{\zeta \rightarrow(\zeta), \quad} \Psi \in \partial K .
$$

Let $* \Psi$ be a conjugate harmonic function on $K^{0}$ for $\Psi$, and set

$$
g(\zeta)=\exp \left[\Psi(\zeta)+i^{*} \Psi(\zeta)\right], \quad \zeta \in K^{0} .
$$

Then $g$ is analytic and bounded on $K^{0}$, and $|g|=\exp (\Psi)$. From (6.1) and (6.2) we obtain

$$
p(\xi) \leqq \lim \inf |g(\zeta)| \leqq \lim \sup |g(\zeta)| \leqq q(\xi),
$$

where $\zeta \in K^{0}$ tends to $\xi \in \partial K$.

Define

$$
V=\left\{(g(\zeta), \zeta / g(\zeta)): \zeta \in K^{0}\right\} \text {. }
$$

Then $V$ is a union of analytic dises in $C^{2}$. The relation (6.3) shows that if $\zeta_{n} \in K^{0}$ tends to $\xi \in \partial K$, then any cluster point $\left(z_{0}, w_{0}\right)$ of the $\left(g\left(\zeta_{n}\right), \zeta_{n} / g\left(\zeta_{n}\right)\right)$ satisfies $z_{0} w_{0}=\xi$ and $p(\xi) \leqq\left|z_{0}\right| \leqq q(\xi)$. In particular, any such cluster point $\left(z_{0}, w_{0}\right)$ belongs to $M_{\zeta}$. It follows that $\bar{V} \backslash V \subset \hat{X}$. By Lemma 3.1, $V \subset \hat{X}$.

For each $\zeta \in K^{0},(g(\zeta), \zeta / g(\zeta))$ belongs to $M_{\zeta}$, so that $F(\hat{X})$ includes $K^{0}$. From Lemma 5.3 and the hypothesis $F(X)=\partial K$, we conclude that $F(\hat{X})=K$. Furthermore, if $\zeta \in K^{0}$, then $Q(\zeta) \geqq|g(\zeta)|=\exp (\Psi(\zeta))$. From Lemma 5.4 it follows that $Q=\exp (\Psi)$ on $K^{0}$, hence on $K$. This proves Theorem 5.2, in the case at hand. In this case, though, we can give a more explicit description of $\hat{X}$.

THEOREM 6.1. Let $X$ be a compact subset of $C^{2}$ that is invariant under the transformations (5.1). Define $F, \Phi, \psi$ and $M_{\zeta}$ as in Sections 5 and 6. Suppose that $F(X)$ forms the boundary $\partial K$ of $a$ polynomially convex subset $K$ of $C$. Let $\Omega$ be a component of $K^{0}$, and let $\zeta \in \Omega$. If $0 \notin \Omega$, then

$$
M_{\zeta}=\{(z, \zeta / z): \Phi(\zeta) \leqq \log |z| \leqq \Psi(\zeta)\}
$$

If $0 \in \Omega$, then

$$
M_{\zeta}=\{(z, \zeta / z): \Phi(\zeta)+G(\zeta, 0) \leqq \log |z| \leqq \Psi(\zeta)\},
$$

where $G(\zeta, 0)$ is the Green's function for $\Omega$ with singularity at 0 .

Proof. In view of Lemma 5.1 and the special case of Theorem 5.2 proved above, we need show only that

$$
\inf \left\{\log |z|:(z, w) \in M_{\zeta}\right\}= \begin{cases}\Phi(\zeta), & \zeta \in \Omega, 0 \notin \Omega, \\ \Phi(\zeta)+G(\zeta, 0), & \zeta \in \Omega, 0 \in \Omega .\end{cases}
$$

The definition of $p$ and the relation $z w=\zeta$ show that 
$\sup \left\{\log |w|:(z, w) \in X \cap M_{\zeta}\right\}=\log |\zeta|-\log p(\zeta), \zeta \in \partial K, \zeta \neq 0$.

Applying the version of Theorem 5.2 already proved, with the variables interchanged, we obtain

$$
\begin{aligned}
\sup \left\{\log |w|:(z, w) \in M_{\zeta}\right\} & =\int[\log |\xi|-\log p(\xi)] d \mu_{\zeta}(\xi) \\
& =\int \log |\xi| d \mu_{\zeta}(\xi)-\Phi(\zeta), \zeta \in \Omega .
\end{aligned}
$$

The relation $z w=\zeta$ then yields

$$
\inf \left\{\log |z|:(z, w) \in M_{\zeta}\right\}=\Phi(\zeta)+\log |\zeta|-\int \log |\xi| d \mu_{\zeta}(\xi),
$$

for $\zeta \in \Omega$. Now $\int \log |\xi| d \mu_{\zeta}(\xi)$ is equal to either $\log |\zeta|$ or $\log |\zeta|-$ $G(\zeta, 0)$, depending on whether $0 \notin \Omega$ or $0 \in \Omega$. That establishes (6.4), and the proof of Theorem 6.1 is complete.

7. An example. As an example, we consider a class of tori which are close to those treated by Wermer [10].

Let $\Gamma$ be any simple closed Jordan curve in $C$ which does not pass through the origin, let $q$ be a positive continuous function on $\Gamma$, and let

$$
X=\{(z, \zeta / z): \zeta \in \Gamma,|z|=q(\zeta)\} .
$$

Then $X$ is a torus that is invariant under the transformations (5.1). In this case, $F(X)=\Gamma$, while $K$ is the union of $\Gamma$ and the bounded component $\Omega$ of $C \backslash \Gamma$. Furthermore, $p=q=Q$ on $\Gamma$. There are two cases that occur.

Suppose $0 \notin \Omega$. Let $\Psi$ be the harmonic extension of $\log q$ to $\Omega$, let $* \Psi$ be a conjugate harmonic function for $\Psi$ on $\Omega$, and set $g(\zeta)=$ $\exp \left(\Psi(\zeta)+i^{*} \Psi(\zeta)\right), \zeta \in \Omega$, as before. Define

$$
V_{\alpha}=\left\{\left(e^{i \alpha} g(\zeta), e^{-i \alpha \zeta / g(\zeta)): \zeta \in \Omega\}, 0 \leqq \alpha<2 \pi} .\right.\right.
$$

Then the $V_{\alpha}$ are disjoint analytic discs whose boundaries lie on $X$, and $\hat{X}$ is the union of $X$ and the $V_{\alpha}$ 's.

On the other hand, suppose $0 \in \Omega$. Define $g$ and the $V_{\alpha}$ 's as above. Also, define an analytic function $h$ on $\Omega$ so that

$$
\log |h(\zeta)|=\log |\zeta|-G(\zeta, 0)-\Psi(\zeta), \zeta \in \Omega,
$$

and set

$$
W_{\alpha}=\left\{\left(e^{\left.\left.-i \alpha \zeta / h(\zeta), e^{i \alpha} h(\zeta)\right): \zeta \in \Omega\right\}, 0 \leqq \alpha<2 \pi} .\right.\right.
$$

The $W_{\alpha}$ are disjoint analytic discs whose boundaries are included in 
$X$. The topological boundary of $\hat{X}$ is the disjoint union of $X$, the $V_{\alpha}$ 's, and the $W_{\alpha}^{\prime} s$. In some sense, $\hat{X}$ is a deformed bidisc.

If the curve $\Gamma$ passes through the origin, one must assume that $q>0$ on $\Gamma \backslash\{0\}$, while

$$
\limsup _{\Gamma \ni \zeta \rightarrow 0} \frac{|q(\zeta)|}{|\zeta|}<\infty
$$

In this case, define $X$ to be the closure of the set of pairs $(z, \zeta / z)$, such that $\zeta \in \Gamma, \zeta \neq 0$, and $|z|=q(\zeta)$. The condition (7.1) guarantees that $X$ is bounded. The polynomial hull $\hat{X}$ of $X$ is the union of $X$, and the analytic discs $V_{\alpha}, 0 \leqq \alpha<2 \pi$, together with an analytic disc or two in the fiber $M_{0}$.

8. Proof of Theorem 5.2: The general case. We return to an arbitrary compact subset $X$ of $C^{2}$ that is invariant under the transformations (5.1). Recall that $K$ is the polynomial hull of $F(X)$. The compact set $Y=F^{-1}(\partial K)$ is also invariant, and $F(Y)=\partial K$. Applying the results of $\S 6$, we find that $F(\hat{Y})=K$. Consequently $F(\hat{X})=K$. To complete the proof of Theorem 5.2, it suffices to establish the identity (5.11).

Set $\log q(\zeta)=-\infty$ on $K \backslash F(X)$, and let $\Omega=\{\zeta \in K: \log q(\zeta)<\Psi(\zeta)\}$. By Theorem 2.1, $\Omega$ is an open subset of $K^{0}$ on which $\Psi$ is harmonic. Let $E$ be the set of $(z, w) \in C^{2}$ such that $\zeta=z w$ belongs to $\Omega$ and $\log |z|=\Psi(\zeta)$. To complete the proof, it will suffice to show that $E \subset \hat{X}$.

Let $\left(z_{0}, w_{0}\right) \in E$, and let $* \Psi$ be the harmonic conjugate function for $\Psi$ defined near $\zeta_{0}=z_{0} w_{0}$ and satisfying $* \Psi\left(\zeta_{0}\right)=\arg z_{0}$. Define $g(\zeta)=\exp \left(\Psi(\zeta)+i^{*} \Psi(\zeta)\right)$ as before. Then as $\zeta$ varies near $\zeta_{0}$, the points $(g(\zeta), \zeta / g(\zeta))$ describe an analytic disc in $E$ which passes through $\left(z_{0}, w_{0}\right)$. Consequently $E$ is a union of analytic discs.

In view of Lemma 3.2 , it suffices now to prove that $\bar{E} \backslash E \subset \hat{X}$.

Suppose $\left(z_{0}, w_{0}\right) \in \bar{E} \backslash E$. Choose $\left(z_{n}, w_{n}\right) \in E$ such that $\left(z_{n}, w_{n}\right)$ converges to $\left(z_{0}, w_{0}\right)$. Then $\zeta_{n}=z_{n} w_{n} \in \Omega$ tends to $\zeta_{0}=z_{0} w_{0}$, and evidently $\zeta_{0} \in \partial \Omega$. Since $\Psi$ is upper semi-continuous, $\log \left|z_{0}\right|=\lim \log \left|z_{n}\right|=$ $\lim \Psi\left(\zeta_{n}\right) \leqq \Psi\left(\zeta_{0}\right)=\log Q\left(\zeta_{0}\right)$. On the other hand, let $u$ be the harmonic extension of $\left.\log q\right|_{\partial K}$ to $K$. Then $u \leqq \Psi$. From (5.5) and the results in the special case already treated,

$$
\log |\zeta|-\log R(\zeta) \leqq u(\zeta), \zeta \in K^{0} .
$$

Using the upper semi-continuity of $R$, we obtain $\log \left|z_{0}\right|=\lim \log \left|z_{n}\right|=$ $\lim \Psi\left(\zeta_{n}\right) \geqq \lim u\left(\zeta_{n}\right) \geqq \log \left|\zeta_{0}\right|-\lim \log R\left(\zeta_{n}\right) \geqq \log \left|\zeta_{0}\right|-\log R\left(\zeta_{0}\right)$. Consequently

$$
\left|\zeta_{0}\right| / R\left(\zeta_{0}\right) \leqq\left|z_{0}\right| \leqq Q\left(\zeta_{0}\right)
$$


From (5.5) and the description of $M_{\zeta_{0}}$ given in $\S 5$, we conclude that $\left(z_{0}, w_{0}\right) \in M_{\zeta_{0}}$. Hence $\bar{E} \backslash E \subset \hat{X}$. The proof is complete.

9. The case in which $\alpha=-m / n$. Let $m$ and $n$ be positive integers that are relatively prime. In this Section, we suppose that $X$ is invariant under the transformations $(z, w) \rightarrow\left(e^{i \theta} z, e^{-i(m / n) \theta} w\right)$. Equivalently, $X$ is invariant under the transformations

$$
(z, w) \longrightarrow\left(e^{i n \theta} z, e^{-i m \theta} w\right),
$$$$
0 \leqq \theta \leqq 2 \pi
$$

The polynomial hull $\hat{X}$ is also invariant under the transformations (9.1).

The description of $\hat{X}$ can be obtained by modifying slightly the discussion of $\S \S 5$ through 8 . In the case at hand, the polynomial map $F$ from $\hat{X}$ to $C$ and the fibers $M_{\zeta}$ are defined by

$$
\begin{array}{cr}
F(z, w)=z^{m} w^{n}, & (z, w) \in \hat{X}, \\
M_{\zeta}=F^{-1}(\{\zeta\}), & \zeta \in C .
\end{array}
$$

The fibers $M_{\zeta}$ are invariant under the transformations (9.1). Again define $Q(\zeta)$ by (5.3) and $R(\zeta)$ by (5.4). If $\zeta \neq 0$, the fiber $M_{\zeta}$ is the annulus or circle given by

$$
M_{\zeta}=\left\{\left(\lambda^{n}, \zeta^{1 / n} / \lambda^{m}\right):|\zeta|^{1 /(m n)} R(\zeta)^{-1 / m} \leqq|\lambda| \leqq Q(\zeta)^{1 / n}\right\} .
$$

Note that the various choices of the $n$th root of $\zeta$ lead to the same set above. The description of $M_{0}$ is idential to (5.7). To describe $\hat{X}$, it again suffices to describe $F(\hat{X})$, and to describe the function $Q$. The final result is almost identical to Theorem 5.2, and so is the proof. We state the result formally, but omit the proof.

THEOREM 9.1. Let $X$ be a compact subset of $C^{2}$ that is invariant under the transformations (9.1). Define $F$ as in (9.2). Then $F(\hat{X})$ coincides with the polynomial hull of $F(X)$. Furthermore, if $Q, q$ and $\Psi$ are as defined in (5.3), (5.8) and (5.10) respectively, then

$$
Q(\zeta)=\exp (\Psi(\zeta))
$$

10. Analytic structure in $\hat{X}$. As a consequence of the description of $\hat{X}$ given above, one can extend Wermer's results on analytic structure in $\hat{X}$ to the compacts sets $X$ treated here.

THEOREM 10.1. Fix $\alpha \leqq 0$, let $X$ be a compact subset of $C^{2}$ that is invariant under the $T_{\theta}$, defined in (0.1). Then every point of $\hat{X} \backslash X$ lies on an analytic disc in $\hat{X}$. Furthermore, $P(X)=C(X)$ if and only if there are no analytic discs in $\hat{X}$. 
Proof. We consider only the case $\alpha=-1$. The other cases are similar.

Let $X$ be a compact subset of $C^{2}$ that is invariant under the transformations (5.1). We will employ the notation of $\S 5$.

Suppose $(z, w) \in \hat{X}$ does not lie on an analytic disc in $\hat{X}$. Let $\zeta=z w$, so that $(z, w) \in M_{\zeta}$. Since $(z, w)$ does not lie in an analytic annulus in $M_{\zeta}$, either $|z|=Q(\zeta)$ or $|w|=R(\zeta)$. Suppose for the sake of definiteness, that $|z|=Q(\zeta)$. If $\zeta \in \Omega$, then $(z, w)$ lies on one of the analytic discs in $\hat{X}$ constructed in the proof of Theorem 5.2 (cf. the third paragraph of $\S 8)$. We conclude that $\zeta \notin \Omega$. Hence $q(\zeta)=$ $Q(\zeta)$. From the definition of $q$ and the invariance of $X$, we conclude that $(z, w) \in X$. That proves the first assertion of the theorem.

If $P(X)=C(X)$, then $\hat{X}=X$, and evidently there are no analytic discs in $X$.

Conversely, suppose that there are no analytic discs in $\hat{X}$. Let $Y=F^{-1}(\partial K)$, as in $\S 8$. Then $Y$ is invariant, $F(Y)=\partial K$, and $F(\hat{Y})=K$. Furthermore, according to the description given in $\S 6$, $\hat{Y} \cap F^{-1}\left(K^{0}\right)$ is a union of analytic discs. We conclude that $K^{0}$ is empty. Hence $K=F(X)$. By Lavrentieff's Theorem, $P(K)=C(K)$. By the theory of sets of antisymmetry [4, Theorem I.13.1], each restriction algebra $\left.P(\hat{X})\right|_{M_{\zeta}}$ is a closed subalgebra of $C\left(M_{\zeta}\right)$, and $P(\hat{X})$ consists of all functions in $C(\hat{X})$ which belong to $\left.P(\hat{X})\right|_{M_{\zeta}}$ for all $\zeta \in K$. Now our hypothesis on $\hat{X}$ shows that each $M_{\zeta}$ is a circle (or a point, if $\zeta=0)$. Furthermore, polynomials in $z^{2} w$ and $z w^{2}$ are dense in $C\left(M_{\zeta}\right)$. Consequently $\left.P(\hat{X})\right|_{M_{\zeta}}=C\left(M_{\zeta}\right)$, and $P(\hat{X})=C(\hat{X})$. In particular, $\hat{X}=X$. The proof is complete.

The following question is open (cf. [5]): Does $P(X)$ consist of precisely those functions $F \in C(\hat{X})$ such that $F$ is analytic on each analytic disc in $\hat{X}$ ?

\section{REFERENCES}

1. L. Carleson, Mergelyan's theorem on uniform polynomial approximation, Math. Scand., 15 (1964), 167-175.

2. K. deLeeuw, A type of convexity in the space of $n$ complex variables, Trans. Amer. Math. Soc., 83 (1956), 193-204.

3. K. deLeeuw, Functions on circular subsets of the space of $n$ complex variables, Duke Math. J., 24 (1957), 415-431.

4. T. Gamelin, Uniform Algebras, Prentice-Hall, Englewood Cliffs, N. J., 1969.

5. — Uniform algebras spanned by Hartogs series, Pacific J. Math., to appear.

6. F. Hartogs, Zur Theorie der analytischen Funktionen mehrerer unabhängiger Veränderlichen, insbesondere über die Darstellung derselben durch Reihen, welche nach Potenzen einer Veränderlichen fortschreiten, Math. Ann., 62 (1906), 1-88.

7. L. Hörmander, An Introduction to Complex Analysis in Several Variables, Van Nostrand, Princeton, N. J., 1966. 
8. M. Tsuji, Potential Theory in Modern Function Theory, Maruzen, Tokyo, 1959.

9. V. S. Vladimirov, Methods of the Theory of Functions of Several Complex Variables, M. I. T. Press, Cambridge, Mass., 1966.

10. J. Wermer, Subharmonicity and hulls, Pacific J. Math., 58 (1975), 283-290.

Received May 13, 1975. This work was supported in part under NSF grant No. GP-42009.

University of CALIFornia, Los ANGeles 


\section{PACIFIC JOURNAL OF MATHEMATICS}

\section{EDITORS}

RICHARD ARENS (Managing Editor) University of California

Los Angeles, California 90024

\section{R. A. BEAUMONT}

University of Washington Seattle, Washington 98105
J. DugundjI Department of Mathematics University of Southern Californı Los Angeles, California 90007

D. Gilbarg AND J. Milgram Stanford University Stanford, California 94305

\section{ASSOCIATE EDITORS}

E. F. BECKENBACH

B. H. NeUMANN

F. WOLF

K. YosHidA

\section{SUPPORTING INSTITUTIONS}

UNIVERSITY OF BRITISH COLUMBIA CALIFORNIA INSTITUTE OF TECHNOLOGY UNIVERSITY OF CALIFORNIA MONTANA STATE UNIVERSITY UNIVERSITY OF NEVADA NEW MEXICO STATE UNIVERSITY OREGON STATE UNIVERSITY UNIVERSITY OF OREGON OSAKA UNIVERSITY

\author{
UNIVERSITY OF SOUTHERN CALIFORNIA \\ STANFORD UNIVERSITY \\ UNIVERSITY OF TOKYO \\ UNIVERSITY OF UTAH \\ WASHINGTON STATE UNIVERSITY \\ UNIVERSITY OF WASHINGTON \\ AMERICAN MATHEMATICAL SOCIETY \\ NAVAL WEAPONS CENTER
}




\section{Pacific Journal of Mathematics}

\section{Vol. 61, No. $1 \quad$ November, 1975}

Jiří Adámek, V. Koubek and Věra Trnková, Sums of Boolean spaces represent every

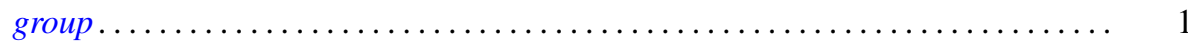

Richard Neal Ball, Full convex l-subgroups and the existence of $a^{*}$-closures of

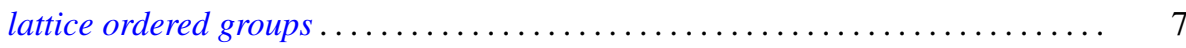

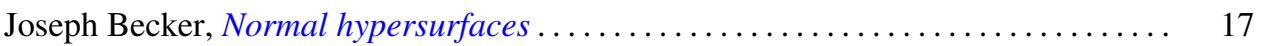

Gerald A. Beer, Starshaped sets and the Hausdorff metric . . . . . . . . . . . . . 21

Dennis Dale Berkey and Alan Cecil Lazer, Linear differential systems with

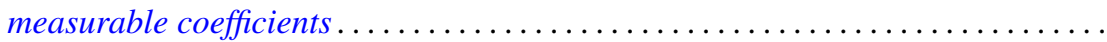

Harald Boehme, Glättungen von Abbildungen 3-dimensionaler

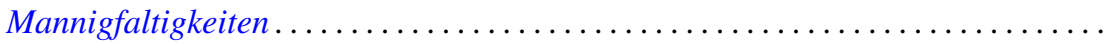

Stephen LaVern Campbell, Linear operators for which $T^{*} T$ and $T+T^{*}$

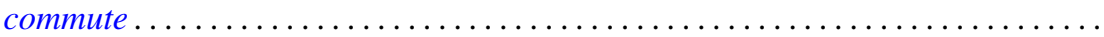

H. P. Dikshit and Arun Kumar, Absolute summability of Fourier series with

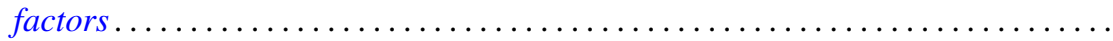

Andrew George Earnest and John Sollion Hsia, Spinor norms of local integral

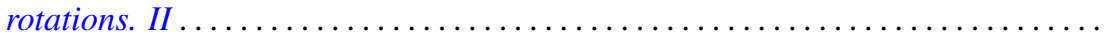

Erik Maurice Ellentuck, Semigroups, Horn sentences and isolic structures .........

Ingrid Fotino, Generalized convolution ring of arithmetic functions . . . . . . . . . . .

Michael Randy Gabel, Lower bounds on the stable range of polynomial rings .......

Fergus John Gaines, Kato-Taussky-Wielandt commutator relations and

characteristic curves

Theodore William Gamelin, The polynomial hulls of certain subsets of $C^{2}$

R. J. Gazik and Darrell Conley Kent, Coarse uniform convergence spaces. . .

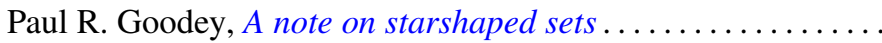

Eloise A. Hamann, On power-invariance

M. Jayachandran and M. Rajagopalan, Scattered compactification for $N \cup\{P\}$. . .

V. Karunakaran, Certain classes of regular univalent functions .

John Cronan Kieffer, A ratio limit theorem for a strongly subadditive set function in a locally compact amenable group .................

Siu Kwong Lo and Harald G. Niederreiter, Banach-Buck measure, density, and uniform distribution in rings of algebraic integers ........

Harold W. Martin, Contractibility of topological spaces onto metric spaces ....

Harold W. Martin, Local connectedness in developable spaces .

A. Meir and John W. Moon, Relations between packing and covering numbers of a tree.

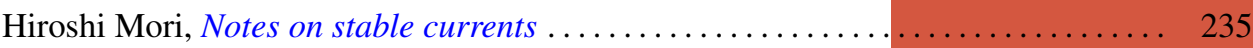

Donald J. Newman and I. J. Schoenberg, Splines and the logarithmic function . . . . 241

M. Ann Piech, Locality of the number of particles operator....

Fred Richman, The constructive theory of $K T$-modules .......

Gerard Sierksma, Carathéodory and Helly-numbers of

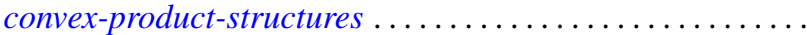

Raymond Earl Smithson, Subcontinuity for multifunctions .... . . 